\title{
First experience imaging short-wave infrared fluorescence in a large animal: indocyanine green angiography of a pig brain
}

Brook K. Byrd

Mikaël Marois

Kenneth M. Tichauer

Dennis J. Wirth

Jennifer Hong

Joseph P. Leonor

Jonathan T. Elliott

Keith D. Paulsen

Scott C. Davis 


\section{First experience imaging short-wave infrared fluorescence in a large animal: indocyanine green angiography of a pig brain}

\author{
Brook K. Byrd, ${ }^{a}$ Mikaël Marois,, ${ }^{a}$ Kenneth M.

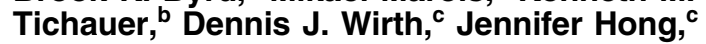 \\ Joseph P. Leonor, ${ }^{a}$ Jonathan T. Elliott, ${ }^{a, c}$ \\ Keith D. Paulsen, ${ }^{a}$ and Scott C. Davis ${ }^{\mathrm{a}, *}$ \\ aDartmouth College, Thayer School of Engineering, Hanover, \\ New Hampshire, United States \\ ${ }^{b}$ Illinois Institute of Technology, Department of Biomedical \\ Engineering, Chicago, Illinois, United States \\ 'Dartmouth-Hitchcock Medical Center, Department of Surgery, \\ Lebanon, New Hampshire, United States
}

\begin{abstract}
The potential to image subsurface fluorescent contrast agents at high spatial resolution has facilitated growing interest in short-wave infrared (SWIR) imaging for biomedical applications. The early but growing literature showing improvements in resolution in small animal models suggests this is indeed the case, yet to date, images from larger animal models that more closely recapitulate humans have not been reported. We report the first imaging of SWIR fluorescence in a large animal model. Specifically, we imaged the vascular kinetics of an indocyanine green (ICG) bolus injection during open craniotomy of a mini-pig using a custom SWIR imaging instrument and a clinical-grade surgical microscope that images ICG in the near-infrared-I (NIR-I) window. Fluorescence images in the SWIR were observed to have higher spatial and contrast resolutions throughout the dynamic sequence, particularly in the smallest vessels. Additionally, vessels beneath a surface pool of blood were readily visualized in the SWIR images yet were obscured in the NIR-I channel. These first-in-large-animal observations represent an important translational step and suggest that SWIR imaging may provide higher spatial and contrast resolution images that are robust to the influence of blood. ๑ The Authors. Published by SPIE under a Creative Commons Attribution 4.0 Unported License. Distribution or reproduction of this work in whole or in part requires full attribution of the original publication, including its DOI. [DOI: 10.1117/1.JBO.24.8.080501]
\end{abstract}

Keywords: short-wave infrared; near-infrared-II window; indocyanine green angiography; medical imaging; fluorescence-guided surgery.

Paper 190145LR received May 7, 2019; accepted for publication Jul. 15, 2019; published online Aug. 10, 2019.

The past five years have witnessed accelerating interest in imaging fluorescent contrast agents in the short-wave infrared (SWIR) wavelength regime for biomedical applications. In living tissue, this regime $(\sim 1000$ to $2000 \mathrm{~nm})$ is characterized by negligible autofluorescence, reduced optical scatter, and lower

*Address all correspondence to Scott C. Davis, E-mail: Scott.C.Davis@ dartmouth.edu or comparable absorption compared to the near-infrared-I (NIR-I) window ( 600 to $900 \mathrm{~nm}) .{ }^{1-3}$ These properties improve both spatial and contrast resolutions, particularly when imaging fluorescence below the surface of tissue, and a reduction in scatter can mitigate confounding background signal from neighboring fluorescent regions within the imaging field. ${ }^{4,5} \mathrm{~A}$ major development for the field was the recent observation that the conventional clinically approved dye indocyanine green (ICG) — in addition to a prominent investigational dye, Licor IRDye800CW (LICOR Biosciences, Lincoln, Nebraska)exhibits measurable fluorescence in the SWIR regime, ${ }^{6,7}$ and follow-up studies indicate that many conventional red/NIR-I fluorophores could be employed for SWIR imaging. ${ }^{8}$ These reports dramatically lower the barrier for preclinical and clinical evaluation of SWIR as a modality for diagnostic and interventional imaging.

The characteristics of SWIR fluorescence propagation in tissue suggest it could be particularly well-suited for imaging the vascular uptake of fluorescent contrast agents, a procedure known as video angiography. In contrast to some targeted molecular imaging strategies that often require detecting low levels of fluorescence in tissue, the technical requirements of angiography are characterized by the ability to resolve relatively small, high-contrast structures (vessels with high concentrations of contrast agent), which plays to the strengths of SWIR imaging in vivo. Current clinical practice relies on ICG angiography carried out in the NIR-I regime to visualize surface and subsurface blood vessels during surgery. ${ }^{9-11}$ This information is used by the surgeon to navigate the tissue, avoiding injury to these critical structures, confirming blood flow, and/or identifying areas for repair. In this context, several published reports of ICG vascular imaging in mouse models have shown that the dye is readily detectable in the SWIR regime and produces images with higher spatial and contrast resolutions in blood vessels as compared to imaging ICG in the NIR-I regime. ${ }^{6,7}$ To date, however, these promising observations have been reported exclusively in mouse models, and images of SWIR fluorescence in model systems that more closely represent humans in size and complexity have not been reported.

Herein, we report the first SWIR fluorescence imaging data in a large animal model. Particularly, we acquired intrasurgical dynamic images of SWIR fluorescence in a pig brain after administration of an ICG bolus. For comparison, the imaging procedure was repeated for the same subject and surgical field using a commercial, clinical-grade surgical microscope that images the NIR-I fluorescence of ICG. Time-lapse visualizations and still frames from both systems were used to make qualitative evaluations and assess spatial and contrast resolution metrics for both SWIR and NIR-I image series.

All animal procedures were conducted in accordance with protocols approved by the Institutional Animal Care and Use Committee at Dartmouth College. A schematic of the procedure timeline is provided in Fig. 1(a). Craniotomy of a 5-month-old Yorkshire mini-pig was completed $30 \mathrm{~min}$ after anesthetization and $\sim 45$ min before initiation of the imaging sessions; one for SWIR fluorescence using a custom SWIR imaging system, and the other for NIR-I fluorescence using a commercial ICG surgical microscope. The two imaging sessions were separated by $1 \mathrm{~h}$ to allow the ICG from the first session to clear.

The SWIR imaging system used in this study consisted of a Newport InstaSpec InGaAs SWIR (InstaSpec, Newport, Irvine, California) camera mounted to a modified Zeiss S1 surgical 


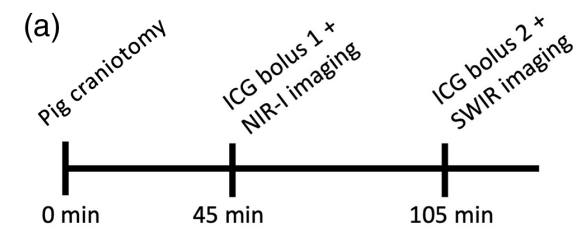

(b)



(c)



Fig. 1 (a) Relative time intervals between craniotomy and the two ICG injections for imaging ICG in the NIR-I and SWIR regimes. (b) Schematic diagram and (c) photograph of the SWIR imaging system used to collect SWIR fluorescence images during open pig craniotomy.

microscope stand (Carl Zeiss AG, Oberkochen, Germany), as shown in Figs. 1(a) and 1(b). The InstaSpec is an InGaAs transferred-electron electron-bombarded charged-coupled device with a $256 \times 1024$ pixel sensor cooled to $-50^{\circ} \mathrm{C}$. Excitation of ICG was provided by a 760-nm laser (CrystaLaser LC, Reno, Nevada) expanded to cover the surgical field at a power intensity of $22 \mathrm{~mW} / \mathrm{cm}^{2}$. While the overall detection field of view was $180 \mathrm{~mm} \times 50 \mathrm{~mm}$ (asymmetric due to the sensor shape), the circular illumination field was $40 \mathrm{~mm}$ in diameter (at $300 \mathrm{~mm}$ from the tissue surface). Fluorescence filtering was provided by an 1100-nm longpass dichroic filter (ThorLabs, Newton, New Jersey) positioned in front of the objective lens. Imaging rates for this system were relatively slow at $2 \mathrm{fps}$. Raw SWIR images were processed by subtracting laser-off dark images and then dividing by the exposure time.

To provide a clinical-grade comparative benchmark, NIR-I fluorescence was imaged using a clinical surgical microscope (Pentero, Carl Zeiss, AG) equipped with the IR800 module designed specifically for ICG angiography. Excitation from this device is provided by a filtered white-light excitation source that illuminates the field at a power intensity of $30 \mathrm{~mW} / \mathrm{cm}^{2}$ and focal distance of $250 \mathrm{~mm}$. The captured image dataset consisted of $430 \times 581$ pixel 8-bit dynamic range data acquired at a frame rate of $30 \mathrm{fps}$.

Each of the two imaging sessions followed the same procedure. First, the imaging system (either the NIR-I or SWIR instrument) was positioned over the surgical field and focused on the exposed brain. Room lights were then dimmed and continuous imaging started shortly before the administration of clinical-grade ICG (Chem-Impex Int'l. Inc., Wood Dale, Illinois) at a $0.07-\mathrm{mg} / \mathrm{kg}$ human equivalent dose into an ear vein. Imaging continued for $5 \mathrm{~min}$ after the bolus administration.

To synchronize the SWIR and NIR-I image series, the NIR-I video recorded by the Pentero was downsampled to match the lower frame rate of the SWIR instrument (total of 166 frames) and the injection times between the two image series were aligned. A commercial motion-correction program, ${ }^{12}$ described extensively elsewhere, ${ }^{13}$ was applied to the NIR-I and SWIR image series individually, and the resulting series combined in a synchronized time-lapse video.
To examine the observed spatial resolution characteristics, cross-sectional profiles of both large and small vessels were extracted from both NIR-I and SWIR postinjection images. Additionally, the dynamic vessel-to-tissue ratio (VTR) was calculated for several selected vessels (for large and small vessels in both NIR-I and SWIR image series).

The time-lapse Video 1 depicts the dynamic uptake of ICG in the brain using NIR-I and SWIR fluorescence from before injection to 5 min after administration. In both channels, the first $10 \mathrm{~s}$ show the fluorescence images prior to ICG administration. The initial exposure time setting for the SWIR camera $(200 \mathrm{~ms})$ was too long for the signal produced by ICG in the vessels, resulting in saturated regions (marked by pink in the video) until the exposure time was reduced to $50 \mathrm{~ms}$, about $30 \mathrm{~s}$ after administration. To avoid confounding effects of saturation, these saturated regions have been excluded from the analyses.

Both videos captured the uptake and distribution of the agent in the vessels. The observed uptake in larger vessels appears similar between the SWIR and NIR-I channels; however, there were stark differences in smaller vascular structures, some of which appeared in the SWIR channel but were not obvious in the NIR-I channel. Overall, the SWIR images appeared sharper throughout the sequence. These observations were examined further by considering selected frames from the dynamic visualization, as provided in Fig. 2. Many of the finer vasculature shown branching from the large vessel in the center of the SWIR images were blurred and/or obscured in the NIR-I images (Fig. 2). Particularly noteworthy observations arose when examining an area of the brain surface covered with a superficial layer of blood [outlined in Figs. 2(a) and 2(b)]. In this region, small vessels were still resolvable in the SWIR images with details comparable to neighboring vessels outside the blood region; however, these vessels were not as clear in the NIR-I image, and the region seemed to suffer from heterogenous reduction in signal compared to other regions of the brain.

Closer inspection of the vasculature in terms of cross-sectional intensity profiles from representative vessels 2-min after ICG-injection (Fig. 3) revealed that while the largest vessel in the field of view (vessel 1) exhibited similar contrast profiles in the SWIR and NIR-I channels, smaller vessels were much more resolvable in the SWIR images. The profiles in Figs. 3(c) and 3(d) show particularly stark differences between the two channels; the SWIR images showed well-resolved vascular features that branch from the large vessel, while the NIR-I profiles were very broad and showed no capacity to resolve these structures.

Finally, we examined local vessel-to-tissue contrast (VTR) for selected vessels in the imaging field throughout the imaging sequence. The vessel and neighboring tissue regions-of-interest (ROIs) used to calculate VTR are shown in Fig. 4 for both NIR-I and SWIR images. Overexposed regions in the early phase of SWIR imaging were excluded. VTR values of both NIR-I and SWIR over time for each vessel analyzed are plotted in Figs. 4(a)-4(d). These plots revealed that after the initial uptake of ICG, local VTR values were relatively stable for both NIR-I and SWIR channels over the 5 min measured. Notably, values of VTR in the SWIR channel were consistently higher than in the NIR-I values. In one example, shown in Fig. 4(b), the VTR measurements for the vessels were $\sim 50 \%$ higher in the SWIR than the NIR-I images. Also notable was the vessel analyzed in Fig. 4(d), which was mostly undetectable in the NIR-I regime, yet produced measurable contrast in the SWIR channel. 

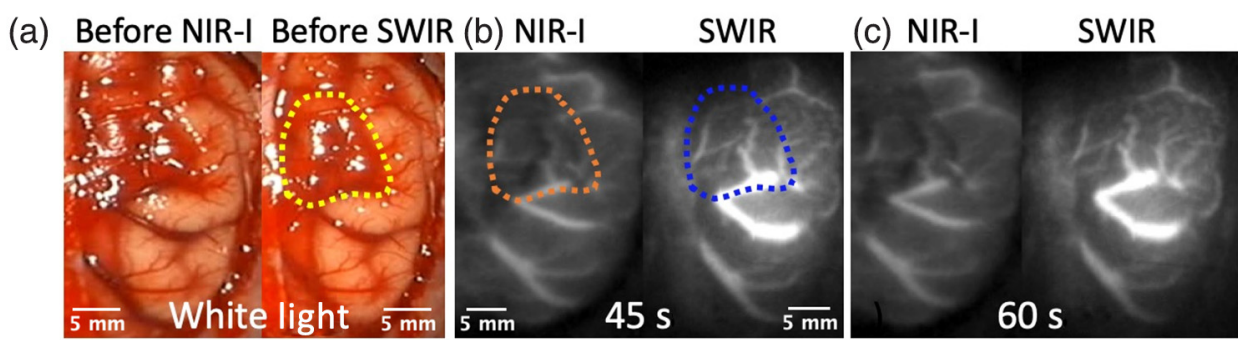

(d)
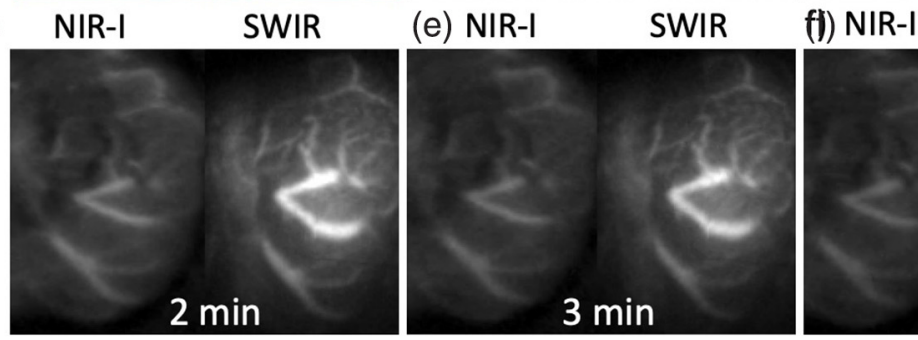

SWIR

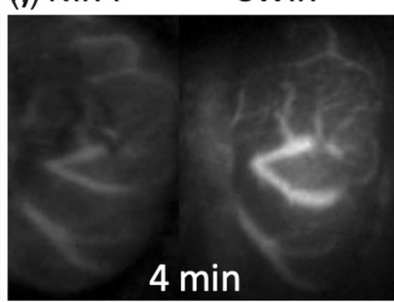

Fig. 2 (a) White-light image taken prior to ICG-bolus injections and (b)-(f) representative frames of the time-lapsed video showing the dynamic uptake of ICG bolus in pig brain using NIR-I (left) and SWIR (right) fluorescence from before injection to 5 min after administration (Video 1, MOV, 697 KB [URL: https://doi.org/10.1117/1.JBO.24.8.080501.1]).

(a) White Light Image

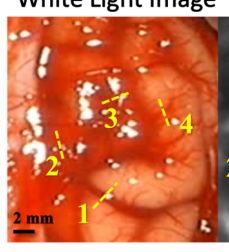

(b)

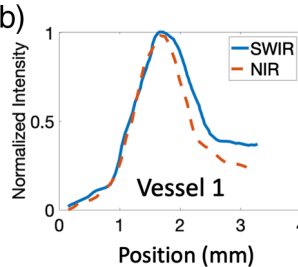

(d)

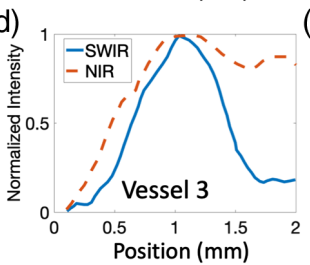

(e) 1

NIR-I Image

SWIR Image

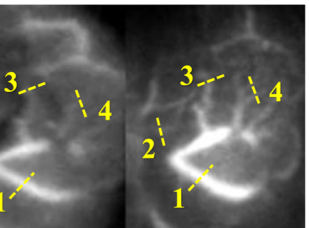

$(c)_{1}$

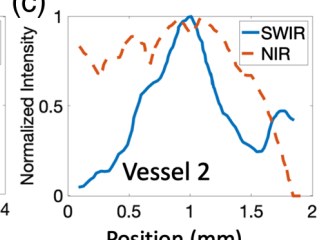



Fig. 3 (a) Locations of profile lines numbered and shown overlaid on white-light, NIR-I, and SWIR images. (b)-(e) Corresponding NIR-I and SWIR intensity profile curves plotted as a function of position.

To our knowledge, this is the first report of SWIR fluorescence in a large animal (nonmouse) model and the first to compare imaging of ICG using SWIR instrument with a clinical NIR-I surgical microscope. Our results indicate that SWIR fluorescence of a human equivalent dose of ICG is readily detectable in this craniotomy model, even when imaging using short exposure times $(50 \mathrm{~ms})$. The vessel structure was well-resolved in the SWIR, and many small vessels that were readily visualized in SWIR images were not visible in the NIR-I images. In the largest vessels, spatial resolution and contrast were similar between the two channels; however, SWIR imaging significantly outperformed the NIR-I system in both spatial and contrast resolutions

in smaller vessels. A particularly notable observation was the stark difference between the two channels in a region of the brain covered by a layer of whole blood. In this region, smaller vessels were still visible in the SWIR images and looked similar to vessels outside the blood region. In contrast, the blood appeared to obscure vessels and produce high signal heterogeneity in the NIR-I images.

These results imply that the superior imaging metrics observed in the SWIR images are a consequence of the favorable optical properties characteristic of the SWIR regime as compared to the NIR-I. However, although the SWIR system outperformed the NIR-I system based on a variety of criteria, we caution against attributing the observations solely to the differences in optical properties in the NIR-I and SWIR regimes. The specifications of each instrument used in this study differ in many respects beyond just the measurement wavelength range. Specifically, the optical components, excitation source, and sensor performance (pixel resolution, bit depth, and sensitivity) are distinct between the two instruments. Although some of the observed features of the two channels cannot be explained by instrumentation differences alone, a rigorous comparison based exclusively on wavelength would require these parameters to be consistent between the two systems. Nonetheless, the results presented here have significant clinical value, particularly considering the confounding issues of blood in the surgical field diminishing fluorescence signals, and the ability of SWIR fluorescence detection to mitigate these effects. As such, these exciting preliminary results establish the strong potential for SWIR-based angiography and instrument-specific performance improvements over a clinical-grade surgical system.

If the results presented herein are translatable to humans, they would have major implications for surgical guidance with fluorescence. The superior resolution, reduced background signal, and robustness to blood pooling observed in the SWIR images represent critical improvements to angiographic and other fluorescence-guided interventions. Efforts to further confirm these results in large animals and clinical cases are underway. 
(a) Visible
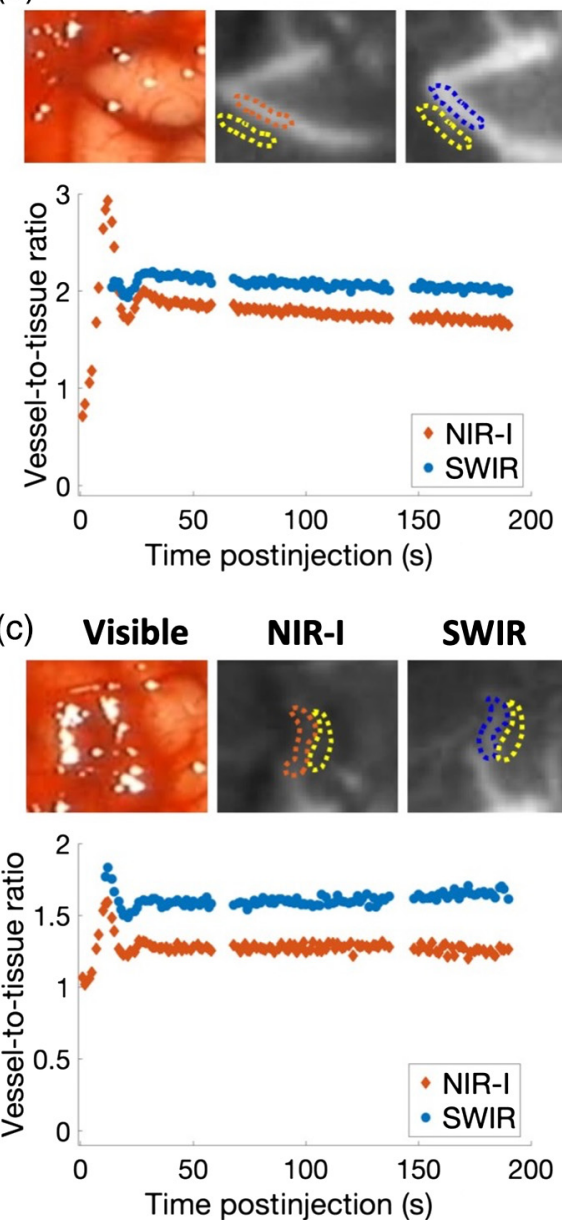

(b) Visible

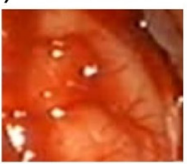

NIR-I
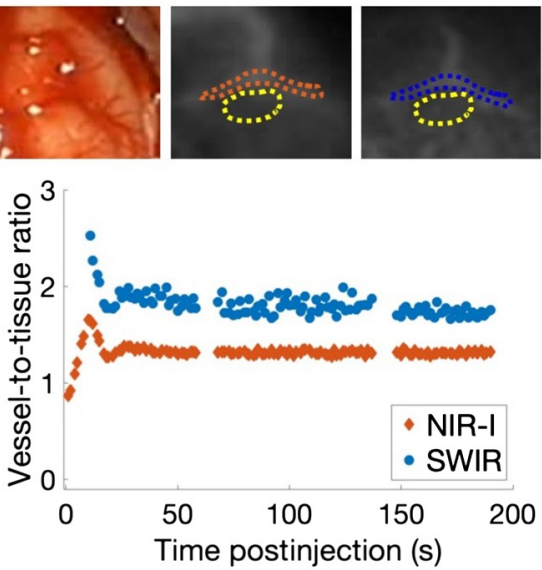

(d)

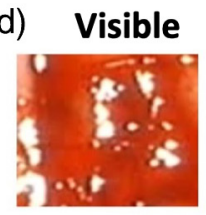

NIR-I

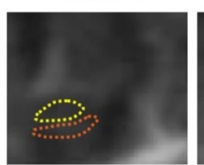

SWIR

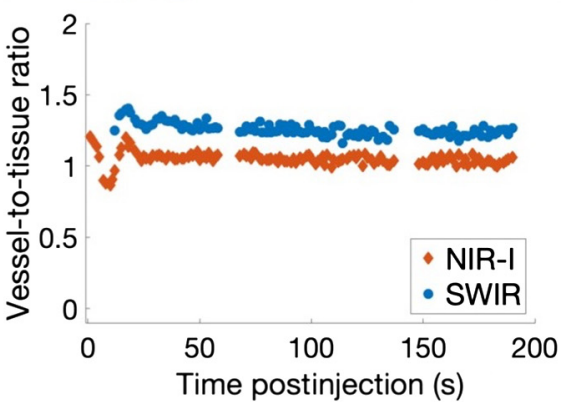

Fig. 4 (a-d) Dynamic VTR values plotted as a function of time with corresponding vessel and tissue ROls outlined above each respective vessel-to-tissue contrast plot.

\section{Disclosures}

The authors have no conflicts of interest to disclose.

\section{Acknowledgments}

The authors thank Physical Sciences Inc. for access to the InstaSpec camera. This work was funded by the National Institutes of Health under Grant Nos. R01CA184354 (S. C. Davis), R01CA188491 (S. C. Davis), R01CA167413 (K. D. Paulsen), and K99CA190890 (J. T. Elliot) and the Hitchcock Foundation under Grant No. HF 250-4083.

\section{References}

1. K. Welsher et al., "A route to brightly fluorescent carbon nanotubes for near-infrared imaging in mice," Nat. Nanotechnol. 4(11), 773-780 (2009).

2. G. Hong et al., "Multifunctional in vivo vascular imaging using nearinfrared II fluorescence," Nat. Med. 18(12), 1841-1846 (2012).

3. D. J. Naczynski et al., "Rare-earth-doped biological composites as in vivo shortwave infrared reporters," Nat. Commun. 4, 2199 (2013).

4. R. H. Wilson et al., "Review of short-wave infrared spectroscopy and imaging methods for biological tissue characterization," J. Biomed. Opt. 20(3), 030901 (2015).
5. J. A. Carr et al., "Absorption by water increases fluorescence image contrast of biological tissue in the shortwave infrared," Proc. Natl. Acad. Sci. U. S. A. 115, 9080-9085 (2018).

6. S. Zhu et al., "Near-infrared-II (NIR-II) bioimaging via off-peak NIR-I fluorescence emission," Theranostics 8(15), 4141-4151 (2018).

7. J. A. Carr et al., "Shortwave infrared fluorescence imaging with the clinically approved near-infrared dye indocyanine green," Proc. Natl. Acad. Sci. U. S. A. 115, 4465-4470 (2018).

8. B. K. Byrd et al., "Characterizing short-wave infrared fluorescence of conventional near-infrared fluorophores," J. Biomed. Opt. 24(3), 035004 (2019).

9. R. Dashti et al., "Microscope-integrated near-infrared indocyanine green videoangiography during surgery of intracranial aneurysms: the Helsinki experience," Surg. Neurol. 71(5), 543-550; discussion 550 (2009).

10. R. Dashti et al., "Microscope integrated indocyanine green videoangiography in cerebrovascular surgery," in Intraoperative Imaging, M. N. Pamir, V. Seifert, and T. Kiris, Eds., pp. 247-250, Springer Vienna, Vienna (2011).

11. V. Kumar et al., "Comparison of efficacy of intraoperative indocyanine green videoangiography in clipping of anterior circulation aneurysms with postoperative digital subtraction angiography," J. Neurosci. Rural Pract. 8(3), 342-345 (2017).

12. Lickenbrock Technologies LLC., Dynamic ICG Tracer Program, software (2006)

13. S. Larkin et al., "Perfusion measures from dynamic ICG scanning laser ophthalmoscopy," Proc. SPIE 7550, 75500H (2010). 